\title{
Sub-harmonic Bifurcation Analysis of Single-Walled Carbon Nanotube Based Mass Sensor
}

\author{
Liangqiang Zhou", Shanshan Liu, Fangqi Chen \\ Department of Mathematics, Nanjing University of Aeronautics and Astronautics, Nanjing, PR China
}

Email address:

zlqrex@sina.com (Liangqiang Zhou)

${ }^{*}$ Corresponding author

\section{To cite this article:}

Liangqiang Zhou, Shanshan Liu, Fangqi Chen. Sub-harmonic Bifurcation Analysis of Single-Walled Carbon Nanotube Based Mass Sensor. Applied and Computational Mathematics. Vol. 5, No. 3, 2016, pp. 97-102. doi: 10.11648/j.acm.20160503.11

Received: March 21, 2016; Accepted: June 4, 2016; Published: June 8, 2016

\begin{abstract}
Bifurcation behaviors are very important for the design of sensors. Using the sub-harmonic Melnikov method, the sub-harmonic bifurcation of single-walled carbon nanotube based mass sensor is investigated in this paper. The parametric conditions for sub-harmonic bifurcation of this system are obtained. It is presented that when the ratio of the excitation amplitude to the damping coefficient crosses a critical value, sub-harmonic bifurcations of $m$ order (odd) can occur. The stability conditions of the bifurcation solution for the system parameters are also obtained by using the affection-angle transformation and average method. The result can provide some guidance for the design of this class of sensors.
\end{abstract}

Keywords: Sub-harmonic Bifurcation, Carbon Nanotube, Sub-harmonic Melnikov Method, Stability

\section{Introduction}

Carbon nanotubes have caused wide public concern over the recent years due to their anisotropic structures and outstanding electrical and mechanical properties since a carbon nanotube was first produced by Iijima in laboratory in 1991 [1] and have been considerable used to fabricate molecular devices [2, 3]. So far, canbon nanotubes have proved to be priming materials for a variety of applications such as flat panel field emission displays [4], nano-electronic devices [5], chemical sensors [6], and batteries [7]. In numerous recent papers, carbon nanotubes were assumed to behave as perfectly straight beams or straight cylindrical shells [8]. However, images taken by transmission electron microscopes for carbon nanotubes show that these tiny structures are not usually straight, but rather have certain degree of curvature or waviness along the nanotubes length $[9,10]$. The curved morphology is due to process-induced waviness during manufacturing processes, in addition to mechanical properties such as low bending stiffness and large aspect ratio.

Nonlinear dynamical behaviors have been investigated widely in recent years. Employing the atomic-scale finite element method, Guo et al [11] studied bending buckling of single-walled carbon nanotubes. It was shown that the appearance of kinks associated with the large deformation nearby reduced the slope of the strain energy curve in the post-buckling stages and hence increases the flexibility of the SWNTs. Wang and Cao [12] studied self-sustained terahertz current oscillation and chaotic dynamics in semiconducting single-walled zigzag carbon nanotubes using the time-dependent drift diffusion equations. The appearance of different nonlinear oscillating modes, including periodic and chaotic, was attributed to the competition between the natural oscillation and the external driving oscillation. Joshi et al [13] investigated nonlinear vibration of a wavy single-walled carbon nanotube based mass sensor. They also studied chaotic responses of single-walled carbon nanotube due to surface deviations with numerical methods [14]. With the Melnikov method and the Preissmann discrete method, Hu et al [15] investigated chaos in an embedded single-walled carbon nanotube. Applying the nonlocal elastic theory and numerical methods, Kuo [16] investigated chaotic vibrations of the single-walled carbon nanotubes on elastic medium. It was shown that the period-three oscillation, the chaos and the period-one oscillation were excited by the different excitation amplitudes. Using the direct perturbation technique, Fang [17] studied chaotic behavior and its control in the single-wall carbon nanotube. With theoretical and numerical approaches, 
Kim and Lee [18] discussed the dynamics of an electrostatically actuated carbon nanotube cantilever. It was presented that high electrostatic excitation could lead to complex nonlinear responses such as softening, multiple stability changes at saddle nodes, or period-doubling bifurcation points in the primary and secondary resonance branches. By using the generalized multi-symplectic method and numerical method, Hu and Deng [19] investigated chaos in an embedded fluid-conveying single-walled carbon nanotube under transverse harmonic load series. It was shown that with the increase in the length of the nanotube and the increase in the number of the transverse load series, the chaotic region respected to the flow velocity decreases obviously. Using the consistent couple stress theory, Fakhrabadi [20] analyzed the small-scale effects on the nonlinear dynamic mechanical and electromechanical behaviors of the carbon nanotubes

In this paper, the sub-harmonic bifurcation of single-walled carbon nanotube based mass sensor is investigated by the sub-harmonic Melnikov method. The parametric conditions for sub-harmonic bifurcation of this system are obtained. The stability conditions of the bifurcation solution are also presented by using the affection-angle transformation and average method.

\section{Formulation of the Problem}

The single-walled CNT based mass sensor investigated in this paper is shown as in Figure 1.

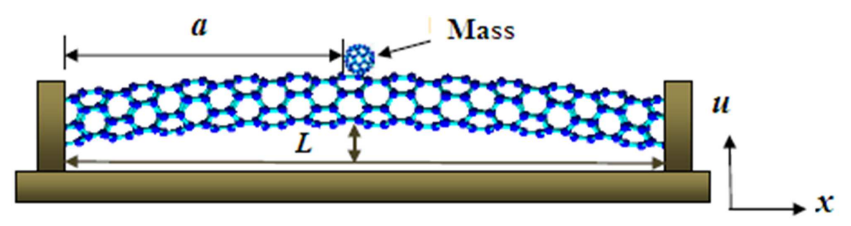

Figure 1. Schematics of a CNT based mass sensor.

Using the Lagrange equation and the Hamiltonian principle, the equation of motion is as follows [13]:

$$
\begin{gathered}
E I \frac{\partial^{4} u}{\partial x^{4}}+\rho A \frac{\partial^{2} u}{\partial t^{2}}+m_{c} \frac{\partial^{2} u}{\partial t^{2}}=F \cos (\omega t) \\
+\frac{E A}{L} \int_{0}^{L}\left[\frac{\partial z}{\partial x} \cdot \frac{\partial u}{\partial x}+\frac{1}{2}\left(\frac{\partial u}{\partial x}\right)^{2}\right] \mathrm{d} x \times\left(\frac{\partial^{2} u}{\partial x^{2}}+\frac{\partial^{2} z}{\partial x^{2}}\right)
\end{gathered}
$$

with the boundary conditions

$$
u(0)=\frac{\mathrm{d} u}{\mathrm{~d} x}(0)=u(L)=\frac{\mathrm{d} u}{\mathrm{~d} x}(L)=0
$$

where $m_{c}$ is the mass attached to the CNT, $\omega$ is the frequency of the transverse load applied due to the attached mass, $E$ is the Young's modulus, $I$ is the second moment of the area, $\rho$ is the density of the nanutobe, $A$ is the cross section area, $F$ is the spatial distribution of the transverse load, $L$ is the length of the nanotube.
Considering the first mode of vibration and implementing the separation of variables technique

$$
u(x, t)=\gamma(x) \theta(t)
$$

Where $\gamma$ and $\theta$ represent the space and domain, respectively.

The first mode shape of a doubly clamped wavy CNT is given as

$$
\gamma(x)=0.816\left(1-\cos \left(\frac{2 \pi x}{L}\right)\right.
$$

Applying the Galerkin approach, the equation of motion of a doubly clamped wavy CNT under condition of primary resonance is as follows [13]:

$$
(1+M) \ddot{\theta}+\alpha \theta+\beta \theta^{2}+\varepsilon \theta^{3}=0.816 \frac{F}{\rho A L^{2}} \cos (\omega t)
$$

where

$$
\begin{gathered}
\alpha=\left(5.33 \frac{E I \pi^{4}}{\rho A L^{4}}+7.74 \frac{\Delta^{2} \pi^{2} E}{\rho L^{4}}\right)=\omega_{0}^{2}, \\
\beta=4.352 \frac{\Delta \pi^{3} E}{\rho L^{4}}, \quad \varepsilon=0.89 \frac{\pi^{4} E}{\rho L^{4}}, \\
M=\frac{m_{c}}{2 \rho A L}
\end{gathered}
$$

Introducing damping in Eq.(5) in order to make the equation of CNT motion more realistic [14] and normalizing the equation using the parameters $t=1 / \omega_{0}$ as a characteristic time and $r=d_{\text {out }}$ as the characteristic length, the non-dimensional form of the equation becomes

$$
(1+M) \ddot{\theta}+2 \xi \dot{\theta}+\theta+\frac{\varepsilon r^{2}}{\alpha} \theta^{3}=\frac{f}{r \alpha} \cos (\omega t)
$$

Considering $\Delta=0$, i.e., the case of straight CNT, then the equation of motion is

$$
(1+M) \ddot{\theta}+2 \xi \dot{\theta}+\theta+\frac{\beta r}{\alpha} \theta^{2}+\frac{\varepsilon r^{2}}{\alpha} \theta^{3}=\frac{f}{r \alpha} \cos (\omega t)
$$

Using the variable transformations

$$
\theta=\frac{x}{\sqrt{\varepsilon r^{2} / \alpha}}, t=\sqrt{1+M} \tau
$$

then Eq.(8) becomes

$$
x^{\prime \prime}+\bar{\zeta} x^{\prime}+x+x^{3}=\bar{f} \cos (\bar{\omega} t)
$$

where ' $=\mathrm{d} x / \mathrm{d} \tau$ is the differential with respect to the dimensionless time $\tau$, and 


$$
\varsigma=\frac{2 \xi}{\sqrt{1+M}}, \bar{f}=\frac{f}{r \alpha}, \tilde{\omega}=\omega \sqrt{1+M}
$$

Assume the damping and excitation terms $\varsigma$ and $\bar{f}$ are small, setting $\varsigma=\varepsilon \tilde{\zeta}, \bar{f}=\varepsilon \tilde{f} \quad(\varepsilon$ is a small parameter), then Eq.(6) can be written as

$$
x^{\prime \prime}+\varepsilon \tilde{\zeta} x^{\prime}+x+x^{3}=\varepsilon \tilde{f} \cos (\tilde{\omega} t)
$$

When $\varepsilon=0$, the unperturbed system is

$$
\left\{\begin{array}{l}
x_{1}^{\prime}=x_{2} \\
x_{2}^{\prime}=-\left(x_{1}+x_{1}^{3}\right)
\end{array}\right.
$$

System (9) is Hamiltonian with Hamiltonian function

$$
H\left(x_{1}, x_{2}\right)=\frac{x_{2}^{2}}{2}+\frac{x_{1}^{2}}{2}+\frac{x_{1}^{4}}{4}
$$

and $(0,0)$ is the unique equilibrium point which is a center; there exist closed periodic orbits around $(0,0)$ with the expressions

$$
\left\{\begin{array}{l}
x_{1 k}(\tau)=\frac{2 k^{2}}{\sqrt{1-2 k^{2}}} \operatorname{cn}\left(\frac{\tau}{\sqrt{1-2 k^{2}}}, k\right) \\
x_{2 k}(\tau)=\frac{\sqrt{2 k^{2}}}{1-2 k^{2}} \operatorname{sn}\left(\frac{\tau}{\sqrt{1-2 k^{2}}}, k\right) \operatorname{dn}\left(\frac{\tau}{\sqrt{1-2 k^{2}}}, k\right)
\end{array}\right.
$$

See Figure 2, where sn, cn, dn are Jacobi elliptic functions. The period of the closed orbit is $T_{k}=4 \sqrt{1-2 k^{2}} K(k)$, where $K(k)$ is the complete elliptic integral of the first kind, and $k$ satisfies

$$
h=\frac{2 k^{2}}{1-2 k^{2}}+\frac{2 k^{4}}{\left(1-2 k^{2}\right)^{2}}
$$

Obviously, when $h \rightarrow \infty$, it follows $k \rightarrow \frac{1}{\sqrt{2}}, T(k) \rightarrow 0$; when $h \rightarrow 0$, it follows that

$$
k \rightarrow 0, T(k) \rightarrow 2 \pi
$$

and

$$
\frac{d T(k)}{d k}<0,
$$

i.e., $T(k)$ is decreases monotonously for $k \in(0,1 / \sqrt{2})$, therefore $T(k) \in(0,2 \pi)$, the smaller the period is, the larger the kinetic energy.

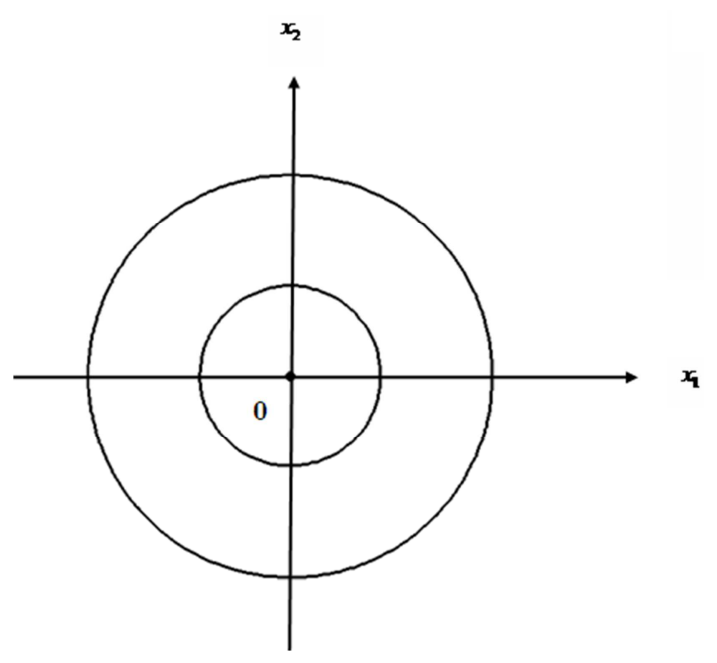

Figure 2. The phase-portrait of system (13).

\section{Sub-harmonic Bifurcations}

In this section, sub-harmonic bifurcations of system (10) are investigated with the sub-harmonic Melnikov method [21]. It can be computed that the sub-harmonic Melnikov function for the periodic orbits (15) satisfying the resonance condition $m T=n T_{k}$ is

$$
\begin{aligned}
M^{m / n}\left(\tau_{0}\right) & =\int_{0}^{m T} x_{2 k}(\tau)\left[-\tilde{\zeta} x_{2 k}(\tau)+\tilde{f} \cos \tilde{\omega}\left(\tau+\tau_{0}\right) \mathrm{d} \tau\right] \\
& =-\tilde{\zeta} I_{1}(m, n)+\tilde{f} I_{2}(m, n) \sin \tilde{\omega} \tau_{0}
\end{aligned}
$$

where

$$
\begin{gathered}
T=\frac{2 \pi}{\tilde{\omega}}, \\
I_{1}(m, n)=\int_{0}^{m T} x_{2 k}^{2}(\tau) \mathrm{d} \tau \\
=\frac{8 m}{3\left(1-2 k^{2}\right)}\left[\frac{1-k^{2}}{1-2 k^{2}} K(k)-E(k)\right], \\
=\left\{\begin{array}{l}
0, n \neq 1 \text { or } m \text { is even; } \\
\frac{2 \sqrt{2}}{\cosh \left(\tilde{\omega} K^{\prime} \sqrt{1-2 k^{2}}\right)}, n=1 \text { and } m \text { is odd }
\end{array}\right.
\end{gathered}
$$

Letting

$$
\begin{aligned}
& R_{1}(m, n)=\frac{I_{1}(m, 1)}{I_{2}(m, 1)}= \\
& \frac{4\left[\left(1-k^{2}\right) K(k)-\left(1-2 k^{2}\right) E(k)\right]}{3 \sqrt{2} \pi \tilde{\omega} \sqrt{\left(1-2 k^{2}\right)^{3}}} \cosh \left(\tilde{\omega} K^{\prime} \sqrt{1-2 k^{2}}\right) \\
= & \frac{8 k}{2 \sqrt{2} \pi^{2} m}\left[\frac{1-k^{2}}{1-2 k^{2}} K(k)-E(k)\right] \cosh \left(\frac{m \pi}{2 K(k)} K^{\prime}(k)\right)
\end{aligned}
$$


When

$$
\frac{\tilde{f}}{\tilde{\zeta}}>R_{1}(m)
$$

there exists a simple zero point for $M^{m / n}\left(\tau_{0}\right)$ for $\tau \in(0,2 \pi)$. Therefore, when the inequality (20) is satisfied, sub-harmonic bifurcations of $m$ (odd) orders for system (12) will occur, consequently periodic vibrations will occur. Denoting $[\tilde{\omega}]$ is the integer part of $\tilde{\omega}$, therefore, when the frequency $\tilde{\omega}$ of the external excitation is fixed, the numbers of sub-harmonic bifurcations of odd orders for the perturbed system is no more than $\frac{1+[\tilde{\omega}]}{2}$.

Remark:

(1) when $k \rightarrow \frac{1}{\sqrt{2}}$, i.e., $\omega \rightarrow \infty, R_{1}(m) \rightarrow \infty$;

(2) when $k \rightarrow 0$, i.e., $\tilde{\omega} \rightarrow m, \lim _{k \rightarrow 0} R_{1}(m)=\left\{\begin{array}{l}0, m=1 \\ \infty, m=3,5,7 \ldots\end{array}\right.$

(3) Further analysis presents that as the increasing of $\frac{\tilde{f}}{\tilde{\zeta}}$ from 0 to $\infty$, no sub-harmonic bifurcations occur for system (12) while $\omega \in(0,1)$; sub-harmonic bifurcation with 1 order (i.e., the periodic is $2 \pi / \tilde{\omega})$ occurs while $\tilde{\omega} \in(1,3)$; sub-harmonic solutions with 1 and 3 orders will occur for $\tilde{\omega} \in(3,5)$, and as the increasing of $\frac{\tilde{f}}{\tilde{\varsigma}}$, sub-harmonic bifurcation with 1 order first occurs, and then sub-harmonic bifurcation with 3 order occurs. Similar results can be obtained for $\tilde{\omega} \in(5,7)$, et al.

\section{Stability of the Sub-harmonic Bifurcations}

In this section, the stability of the sub-harmonic bifurcations is investigated with the affection-angle transformation and the second order average method.

Using the affection-angle transformation

$$
\left\{\begin{array}{l}
I=I\left(x_{1}, x_{2}\right) \\
v=v\left(x_{1}, x_{2}\right)
\end{array}\right.
$$

System (8) can be written as

$$
\left\{\begin{array}{l}
I^{\prime}=0 \\
v^{\prime}=\Omega(I)
\end{array}\right.
$$

where

$$
\Omega(I)=\frac{\partial H}{\partial I}=\frac{2 \pi}{T_{R}}
$$

$$
\left\{\begin{array}{l}
I^{\prime}=\varepsilon \frac{\partial I}{\partial x_{2}}\left(\tilde{f} \cos (\tilde{\omega} \tau)-\tilde{\zeta} x_{2}\right) \triangleq \varepsilon F(I, v, \tau) \\
v^{\prime}=\Omega(\mathrm{I})+\varepsilon \frac{\partial v}{\partial x_{2}}\left(\tilde{f} \cos (\tilde{\omega} \tau)-\tilde{\zeta} x_{2}\right) \triangleq \varepsilon G(I, v, \tau)
\end{array}\right.
$$

Consider the perturbation of the sub-harmonic orbit with period $T_{k}=2 \pi m / n,(m=1,3,5 \ldots)$

$$
\left\{\begin{array}{l}
I=I^{k}+\sqrt{\varepsilon} h \\
v=\Omega\left(I^{k}\right) t+\phi \triangleq \Omega^{k} t+\phi
\end{array}\right.
$$

where $I^{k}$ is the affection variable. Instituting (21) into (20), and expanding (16) to $o(\varepsilon)$, one can obtain that

$$
\begin{aligned}
& h^{\prime}=\frac{\sqrt{\varepsilon}}{\Omega\left(I^{k}\right)} x_{2 k}\left(t-\frac{\phi}{\Omega^{k}}\right)\left[\tilde{f} \cos \tilde{\omega} t-\tilde{\varsigma} x_{2 k}\left(t-\frac{\phi}{\Omega^{k}}\right)\right] \\
& +\varepsilon \frac{\partial}{\partial I} F\left(I^{k}, \Omega^{k} t+\phi, t\right) h+O\left(\varepsilon^{3 / 2}\right)
\end{aligned}
$$

$$
\begin{aligned}
& \phi^{\prime}=\sqrt{\varepsilon} \frac{\mathrm{d} \Omega}{\mathrm{d} I}\left(I^{k}\right) h+\varepsilon\left[\frac{\frac{\mathrm{d}^{2} \Omega}{\mathrm{d}^{2} I}\left(I^{k}\right) h^{2}}{2}\right. \\
& \left.+\mathrm{G}\left(I^{k}, \Omega^{k} t+\phi, t\right)\right]+O\left(\varepsilon^{3 / 2}\right)
\end{aligned}
$$

Denote

$$
\begin{gathered}
\frac{1}{\Omega\left(I^{k}\right)} x_{2 k}\left(t-\frac{\phi}{\Omega^{k}}\right)\left[\tilde{f} \cos \tilde{\omega} t-\tilde{\zeta} x_{2 k}\left(t-\frac{\phi}{\Omega^{k}}\right)\right] \\
=M^{m}\left(\frac{\phi}{\Omega^{k}}\right)+\tilde{F}(\phi, t),
\end{gathered}
$$

where $M^{m}\left(\phi / \Omega^{k}\right)$ is the Melnikov integration of system (12) along the sub-harmonic orbit, the period of $\tilde{F}(\phi, t)$ is $T=2 \pi / \tilde{\omega}$, and average value of $\tilde{F}(\phi, t)$ is 0 .

Using the average transformation

$$
h \rightarrow \bar{h}+\sqrt{\varepsilon} \int \tilde{F}(\phi, t) d t, \quad \phi \rightarrow \bar{\phi}
$$

Eq.(26) can be written as

$$
\begin{aligned}
& h^{\prime}=\frac{\varepsilon}{2 \pi} M^{m}\left(\frac{\bar{\phi}}{\Omega^{k}}\right)+\varepsilon\left[\frac{\partial}{\partial I} F\left(I^{k}, \Omega^{k} t+\phi, t\right)\right. \\
& \left.+\frac{\mathrm{d} \Omega}{\mathrm{d} I}\left(I^{k}\right) \bar{h} \frac{\partial}{\partial \phi} \int \bar{F} d t\right]+O\left(\varepsilon^{3 / 2}\right) \\
& \phi^{\prime}=\sqrt{\varepsilon} \frac{d \Omega}{d I}\left(I^{k}\right) \bar{h}+\varepsilon\left[\frac{\frac{d^{2} \Omega}{d^{2} I}\left(I^{k}\right) h^{2}}{2}\right. \\
& \left.\left.+\mathrm{G}\left(I^{k}, \Omega^{k} t+\phi, t\right)\right]+\frac{d \Omega}{d I}\left(I^{k}\right) \int \bar{F} d t\right]+O\left(\varepsilon^{3 / 2}\right)
\end{aligned}
$$

and system (12) can be written as 
The mean value of $\int \tilde{F} d t$ and $\frac{\partial}{\partial \phi} \int \tilde{F} d t$ is also 0 since the mean value of $\tilde{F}$ is 0 .

Applying the average theory to (25), using the transformation $t \rightarrow \sqrt{\varepsilon} t$, and denoting $(\bar{h}, \bar{\phi})$ as $(h, \phi)$, one can obtain that

$$
\begin{gathered}
h^{\prime}=\frac{1}{2 \pi} M^{m}\left(\frac{\phi}{\Omega^{k}}\right)+\sqrt{\varepsilon} \overline{F^{\prime}(\phi) h}+O(\varepsilon) \\
\phi^{\prime}=\frac{\mathrm{d} \Omega}{\mathrm{d} I}\left(I^{k}\right) h+\sqrt{\varepsilon}\left[\frac{\frac{\mathrm{d}^{2} \Omega}{\mathrm{d}^{2} I}\left(I^{k}\right) h^{2}}{2}+\overline{G(\phi)}\right]+O(\varepsilon)
\end{gathered}
$$

where

$$
\begin{gathered}
\overline{F^{\prime}(\phi)}=\frac{1}{m T} \int_{0}^{m T} \frac{\partial}{\partial I} F\left(I^{k}, \Omega^{k} t+\phi, t\right) \mathrm{d} t \\
\overline{G^{\prime}(\phi)}=\frac{1}{m T} \int_{0}^{m T} G\left(I^{k}, \Omega^{k} t+\phi, t\right) \mathrm{d} t
\end{gathered}
$$

Then the hyperbolic or elliptic fixed points of system (29) are corresponding to the sub-harmonic orbits of $m$ orders for system (12).

Noting that in the affection-angle transformation, $\frac{\partial x_{1}}{\partial I}=-\frac{\partial v}{\partial x_{2}}$ is hold, so we have

$$
\begin{aligned}
& \overline{F^{\prime}(\phi)}=\frac{\tilde{\omega}}{2 \pi m} \int_{0}^{\frac{2 \pi m}{\tilde{\omega}}} \frac{\partial}{\partial I}\left\{\frac{1}{\Omega(\mathrm{I})} x_{2 k}\left(\mathrm{t}-\frac{\phi}{\Omega^{k}}\right)\right. \\
& \left.\left[\tilde{f} \cos \tilde{\omega} t-\tilde{\varsigma} x_{2 k}\left(t-\frac{\phi}{\Omega^{k}}\right)\right]\right\}\left.\right|_{I=I^{k}} \mathrm{~d} t \\
& \overline{G(\phi)}=\frac{\tilde{\omega}}{2 \pi m} \int_{0}^{\frac{2 \pi m}{\tilde{\omega}}}-\frac{\partial x_{1 k}}{\partial I} \\
& \left.\left[\tilde{f} \cos \tilde{\omega} \mathrm{t}-\tilde{\varsigma} x_{2 k}\left(t-\frac{\phi}{\Omega^{k}}\right)\right]\right\}\left.\right|_{I=I^{k}} \mathrm{~d} t
\end{aligned}
$$

When $\frac{\mathrm{d} \Omega}{\mathrm{d} t}<\infty, \mathcal{E}$ is small, the stability condition of sub-harmonic orbits can be determined by the trance of the linearization of system (29). The linearization matrix of system (29) is

$$
A=\left(\begin{array}{cc}
\sqrt{\varepsilon} \overline{F^{\prime}(\phi)} & \frac{1}{2 \pi} \frac{\partial}{\partial \phi} M^{m}\left(\phi / \Omega^{k}\right) \\
\frac{\mathrm{d}}{\mathrm{d} \phi}\left(I^{k}\right)+\sqrt{\varepsilon} \frac{d^{2}}{d I^{2}}\left(I^{k}\right) h & \sqrt{\varepsilon} \frac{\partial}{\partial \phi} \overline{G(\phi)}
\end{array}\right)
$$

The trance of $A$ is

$$
\operatorname{trace} A=\sqrt{\varepsilon} \overline{F^{\prime}(\phi)}+\sqrt{\varepsilon} \frac{\partial}{\partial \phi} \overline{\mathrm{G}(\phi)}
$$

When $\operatorname{trace} A>0$, the sub-harmonic orbit is saddle or source type; while trace $A<0$, it is saddle or sink.

Using the following results:

$$
\begin{aligned}
& \frac{\partial \Omega}{\partial k}=\frac{\pi k}{\left(1-2 k^{2}\right)^{3 / 2} K}-\frac{\pi\left(E-\left(1-k^{2}\right) K\right)}{2 K^{2} k \sqrt{1-2 k^{2}}\left(1-k^{2}\right)} \\
& \frac{\mathrm{d} K(k)}{\mathrm{d} k}=\frac{E(k)}{k\left(1-k^{2}\right)}-\frac{K(k)}{k}, \frac{\mathrm{d} E(k)}{\mathrm{d} k}=\frac{E(k)-K(k)}{k} \\
& \frac{\mathrm{d} K(k)}{\mathrm{d} k}=-\frac{E^{\prime}-k^{2} K^{\prime}}{k\left(1-k^{2}\right)} \\
& \int_{0}^{\frac{2 \pi m}{\tilde{\omega}}} \frac{1}{\Omega} x_{2 k}\left(t-\frac{\phi}{\Omega^{k}}\right) \cos \tilde{\omega} t \mathrm{~d} t=\frac{1}{m} I_{2}(m, 1) \cos \left(\tilde{\omega} \frac{\phi}{\Omega^{k}}\right) \\
& \frac{\partial}{\partial k} I_{1}(m, 1)=\frac{8 k(2 E(k)-K(k))}{3\left(1-2 k^{2}\right)^{2}} \\
& \frac{\partial}{\partial k} I_{2}(m, 1)=\frac{\sqrt{2} m \pi \sinh \left(\frac{m \pi K^{\prime}}{2 K}\right)}{\cosh ^{2}\left(\frac{m \pi K^{\prime}}{2 K}\right)} \\
& \frac{\left(E^{\prime}-k^{2} K^{\prime}\right) K+\left(E-\left(1-k^{2}\right) K\right) K^{\prime}}{k\left(1-k^{2}\right) K^{2}}
\end{aligned}
$$

Substituting (35)-(38) into (34), the stability conditions for the sub-harmonic conditions can be obtained.

The result can provide some guidance for the design of this class of sensors. For example, while design the sensor, we should chose the system parameters suitable, such that they belong to the stable region, otherwise the periodic solutions are unstable and chaotic motions may occur, which can bring some damage to the sensors.

\section{Conclusions}

The sub-harmonic bifurcation of single-walled carbon nanotube based mass sensor is investigated with the sub-harmonic Melnikov method in this paper. The parametric conditions for sub-harmonic bifurcation of this system are obtained. It is shown that when the ratio of the excitation amplitude to the damping coefficient crosses a critical value, sub-harmonic bifurcations of $m$ order (odd) can occur. The stability conditions of the bifurcation solution for the system parameters are also obtained by using the affection-angle transformation and average method. It provides some inspiration and guidance for the analysis and dynamic design of this class of system. For example, we should chose the system parameters suitable so that the periodic solutions are stable; otherwise chaotic motions may occur and damages to the sensors may happen. 


\section{Acknowledgements}

The project was supported by National Science Foundation of China (11202095, 11572148), China Postdoctoral Foundation (2013T60531), and the Doctoral Program of Higher Education of China (20133218110025).

\section{References}

[1] S. Iijima, Helical microtubules of graphitic carbon. Nature 354(6348), 56-58 (1991).

[2] S. J. Tans, A. R. M. Verschueren, C Dekker, Roomtemperature transistor based on a single carbon nanotube. Nature 393(6680), 49-52 (1998).

[3] R. Martel, T. Schmidt, H. R. Shea, T. Hertel, P. Avouris, Single- and multi-wall carbon nanotube field-effect transistors. Appl. Phys. Lett. 73(17), 2447-2449 (1998).

[4] W. A. De Heer, W. S. Bacsa, A. Châtelain, T. Gerfin, R. Humphrey-Baker, L. F orro, D. Ugarte, Aligned carbon nanotube films: production and optical and electronic properties. Science 268(5212), 845-847 (1995).

[5] A. Zettl, xtreme oxygen sensitivity of electronic properties of carbon nanotubes. Science 287(5459), 1801-1804 (2000).

[6] J. Kong, N. R. Franklin, C. Zhou, M. G. Chapline, S. Peng, K. Cho, H Dai, Nanotube molecular wires as chemical sensors. Science 287(5453), 622-625 (2000).

[7] G. Che, B. B. Lakshmi, E. R. Fisher, C. R. Martin. Carbon nanotubule membranes for electrochemical energy storage and production. Nature 393(6683), 346-349 (1998).

[8] R. F. Gibson, O. A. Emmanuel, F. W. Yuan. Vibrations of carbon nanotubes and their composites: a review. Compos Sci Technol 2007; 67(1): 1-28.

[9] D, Qian. E. C. Dickey. R. Andrews, T. Rantell. Load transfer and deformation mechanisms in carbon nanotube-polystyrene composites. Appl Phys Lett 2000; 76(20): 2868-70.

[10] Z. L. Wang. P. Poncharal. W. A. Heer. Nanomeasurements of individual carbon nanotubes by in situ TEM. Pure Appl Chem 2000; 72(1-2): 209-19.
[11] X. Guo, A. Y. T. Leung, X. Q. He, et al. Bending buckling of single-walled carbon nanotubes by atomic-scale finite element, Composites: Part B Engineering, 2008; 39(1): 202-208.

[12] C. Wang, J. C. Cao, Terahertz generation and chaotic dynamics in single-walled zigzag carbon nanotubes, Chaos, 2009; 19: 033136.

[13] A. Y. Joshi, S. C. Sharma, S. P. Harma, Nonlinear dynamic analysis of single-walled carbon nanotube based mass sensor, Journal of Nanotechnology in Engineering and Medicine, 2011; 2: 041008 .

[14] A. Y. Joshi, S. C. Sharma, S. P. Harsha, Chaotic responses analysis of single-walled carbon nanotube due to surface deviations, NANO: Brief Reports and Reviews, 2012; 7(2): 1250008 .

[15] W. P. Hu, Z. C. Deng, B. Wang. et al. Chaos in an embedded single-walled carbon nanotube, Nonlinear Dynamics, 2013; 73(1): 389-398.

[16] Y. L. Kuo, Chaotic Analysis of the Geometrically Nonlinear Nonlocal Elastic Single-Walled Carbon Nanotubes on Elastic Medium, Journal of Nanoscience and Nanotechnology, 2014, 14(3): 2352-2360.

[17] J. S. Fang, Chaotic behavior and its control in the single-wall carbon nanotube, International Journal of Modern Physics B, 2014, 28: 1450005 .

[18] I. K. Kim, S. I. Lee, Nonlinear resonances of a single-wall carbon nanotube cantilever, Physica E, 2015, 67: 159-167.

[19] W. P. Hu, Z. C. Deng, Chaos in embedded fluid-conveying single-walled carbon nanotube under transverse harmonic load series, Nonlinear Dynamics, 2015, 79(1): 325-333.

[20] M. M. S. Fakhrabadi, Prediction of small-scale effects on nonlinear dynamic behaviors of carbon nanotube-based nano-resonators using consistent couple stress theory, Composites Part B, 2016, 88(1): 26-35.

[21] J. Guckenheimer, P. Holmes, Nonlinear Oscillations, Dynamical Systems, and Bifurcaions of Vector Fields, Springer, New York, 1997, pp. 195. 